\title{
Autorisation de mise sur le marché du Ca-DTPA
}

\author{
L. GRAPPIN ${ }^{1}$, P. BÉRARD ${ }^{2}$
}

Depuis plus de 40 ans, la forme injectable du Ca-DTPA est utilisée dans le traitement des contaminations internes (par inhalation, plaie ou passage percutané) aux transuraniens, principalement le plutonium, l' américium et le curium. Le Ca-DTPA agit par chélation, il favorise l'excrétion urinaire des radioéléments plutonium, américium et curium et en limite le passage vers les organes de dépôt. Ce traitement n'est pas indiqué dans le cas d'exposition à l'uranium ou au neptunium.

Le pentétate de calcium trisodique est le sel de sodium du diéthylènetriaminepentaacétate calcique. Il est également connu sous le nom de trisodium calcium diéthylènetriaminepentaacétate et est noté Ca-DTPA. Sa formule moléculaire est $\mathrm{Na}_{3} \mathrm{CaC}_{14} \mathrm{H}_{18} \mathrm{~N}_{3} \mathrm{O}_{10}$ et sa masse moléculaire est de 497,4 daltons.

Le Ca-DTPA est fabriqué en France par la pharmacie centrale des armées qui a déposé auprès de l'Afssaps (Agence française de sécurité des produits de santé) un dossier de demande d'autorisation de mise sur le marché. Cette AMM a été accordée le 12 février 2008.

\section{Mode d'administration}

Le Ca-DTPA $250 \mathrm{mg} / \mathrm{mL}$, solution injectable doit être administré en injection intraveineuse lente ou par perfusion d'un quart d'heure environ.

\section{Indications}

L’indication majeure est le traitement de la plaie contaminée.

Le traitement est d'autant plus efficace qu'il est administré précocement, il doit être débuté dès que possible en cas de suspicion de contamination.

Pour les inhalations, l'efficacité est plus inconstante, le traitement initial mérite cependant d'être mis en place selon le niveau d'exposition.

${ }^{1}$ CEA Cadarache, SST, Bâtiment 102, 13108 Saint-Paul-Lez-Durance, France.

${ }^{2}$ CEA Saclay, LABM, Bâtiment 601, 91191 Gif-sur-Yvette Cedex, France. 
Les résultats radiotoxicologiques des examens urinaires guident ensuite la poursuite du traitement.

\section{Posologie}

\section{Voie intraveineuse}

- La posologie recommandée chez l'adulte et chez l'adolescent est de 0,5 g par jour (soit 1/2 ampoule), sans dépasser $1 \mathrm{~g}$ par jour ;

- chez les enfants de moins de 12 ans, la posologie est déterminée en fonction de la masse corporelle $(14 \mathrm{mg} / \mathrm{kg})$ sans dépasser $0,5 \mathrm{~g}$ par jour.

En cas de nécessité de traitement prolongé, le schéma suivant peut être proposé :

- 1 injection par jour pendant 3 à 5 jours ;

- 2 à 3 injections par semaine pendant 3 semaines ;

- 1 injection par semaine pendant 3 mois ;

- à poursuivre au-delà de 3 mois, en fonction des résultats de la mesure de l'excrétion urinaire. La posologie peut alors être réduite à $0,25 \mathrm{~g}$ par jour.

\section{Voie cutanée}

En traitement local, cette voie peut être utilisée en complément de la voie intraveineuse :

- une à plusieurs ampoules versées directement sur la peau saine, en associant les autres méthodes de décontamination ;

- une à plusieurs ampoules sur les plaies contaminées.

\section{Contre-indication}

Il n'y a pas de contre-indication en dehors d'une réaction allergique à une précédente injection.

\section{Titulaire de l'AMM, exploitant et fabricant du Ca-DTPA}

Pharmacie centrale des armées, BP 04, 45998 Orléans Armées, France

Dénomination de la spécialité: CIS : 69580203 - Date : 12 février 2008

Classe pharmacothérapeutique : antidote — Code ATC : V03AB

\section{POUR EN SAVOIR PLUS}

Grappin L., Bérard Ph., Ménétrier F., Carbone L., Castagnet X., Le Goff J.P., Méron M.O., Piechowski J. (2007) Treatment of actinide exposures: A review of Ca-DTPA injections inside CEAAREVA plants, Radioprotection 42, 163-196.

Lien relatif à l'autorisation de mise sur le marché du Ca-DTPA : http://afssaps-prd.afssaps.fr/ php/ecodex/extrait.php?specid=69580203 